\title{
OBITUARIO
}

\section{Obituario, Dr. Bernardo Reyes Leal (Q.E.P.D.)}

El pasado 21 de enero de 2021 registramos con gran pesar el fallecimiento de nuestro profesor y mentor, el Dr. Bernardo Reyes Leal, endocrinólogo, miembro honorario y expresidente de la Asociación Colombiana de Endocrinología, Diabetes y Metabolismo (ACE), profesor emérito de la facultad de Medicina de la Universidad Nacional, fundador junto con otros reconocidos colegas de la Asociación Colombiana de Medicina Interna (ACMI), y desde su regreso a Colombia en el año de 1962, impulsor y colaborador incansable en el progreso y la evolución de nuestra Asociación, en la que además creó y promovió sus publicaciones, incluida esta revista.

El Dr. Bernardo Reyes Leal realizó sus estudios de medicina en Francia y a su regreso fue seleccionado dentro de un grupo de profesores de la Universidad Nacional para complementar sus estudios de endocrinología en la Universidad de Ann Arbor en Michigan, bajo la tutoría del Dr. Jerome W. Conn, descubridor del hiperaldosteronismo primario. A su regreso a Colombia y como profesor titular de Endocrinología en el Hospital San Juan de Dios de Bogotá, se dedicó al desarrollo científico y académico de la especialidad, dando un énfasis incomparable a la investigación y siendo promotor del desarrollo de la medicina nuclear en el Hospital y posteriormente del laboratorio hormonal, incluido el montaje del radioinmu- noensayo, que permitió las primeras mediciones de hormonas por este método en el país.

Liderando el mejor nivel académico en el área de la endocrinología, inició la creación de los programas de especialización en nuestro país y en la década de los ochenta logró el primer programa de especialización en Endocrinología en la Universidad Nacional y apoyando desde la ACE la creación de otros programas en las universidades del Rosario, Militar y Javeriana, que consolidaron el semillero de endocrinólogos con el que hoy cuenta Colombia.

Dr. Bernardo Reyes Leal, lo recordaremos por siempre, maestro de maestros, investigador inquieto de extraordinaria inteligencia, visionario, totalmente adelantado a su tiempo, siempre inquietando a los que fuimos sus alumnos con propuestas que resultaban temerarias y que hoy, 40 años después, son dogmas de la endocrinología.

Gracias, querido maestro.

Amanda Páez Talero

Médica Internista, Endocrinóloga

Expresidente y Miembro Honorario de la Asociación Colombiana de

Endocrinología, Diabetes y Metabolismo 


\section{SANOFI}

\section{AYUDE A SUS PACIENTES A LOGRAR LA META DE HbA1c}

$\mathbf{7 4} \%$ de los pacientes que venían descontrolados con ADOs, alcanzaron la meta de $\mathrm{HbAlc}$ con una sola inyección al día de SOLIQUA ${ }^{\boxplus 1}$.

SOLIQUA ${ }^{\circledR}$ reduce en promedio $-2,9 \%$ * de $\mathrm{HbAlc}$ en el sub-grupo de pacientes con $\mathrm{HbAlc}>9 \%$ al inicio del tratamiento?

*Promedio de reducción en pacientes tratados previamente con ADOs con HbAlC > 9\%; el promedio de reducción de HbAlc del total de pacientes descontrolados tratados previamente con ADOs fue $-1,6 \%$.

Conozca más sobre SOLIQUA ${ }^{\boxplus}$ descargando

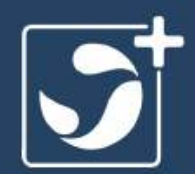

SANOFIMED+

Google play

Capp Store

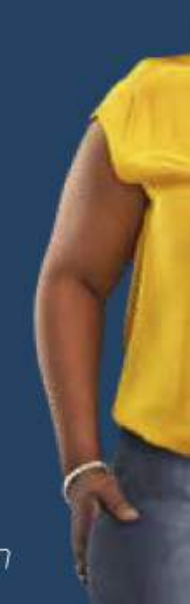

